\title{
Bokeh Mirror Alignment for Cherenkov Telescopes
}

\author{
M. L. Ahnen ${ }^{\mathrm{a}}$, D. Baack ${ }^{\mathrm{d}}$, M. Balbo ${ }^{\mathrm{b}}$, M. Bergmann ${ }^{\mathrm{c}}$, A. Biland ${ }^{\mathrm{a}}$, M. Blank ${ }^{\mathrm{c}}$, \\ T. Bretz ${ }^{\mathrm{a}}$, K. A. Bruegge ${ }^{\mathrm{d}}$, J. Buss ${ }^{\mathrm{d}}$, M. Domke ${ }^{\mathrm{d}}$, D. Dorner ${ }^{\mathrm{c}}$, S. Einecke ${ }^{\mathrm{d}}$, \\ C. Hempfling ${ }^{\mathrm{c}}$, D. Hildebrand ${ }^{\mathrm{a}}$, G. Hughes ${ }^{\mathrm{a}}$, W. Lustermann ${ }^{\mathrm{a}}$, K. Mannheim ${ }^{\mathrm{c}}$, \\ S. A. Mueller a, D. Neise ${ }^{\mathrm{a}}$, A. Neronov ${ }^{\mathrm{b}}$, M. Noethe ${ }^{\mathrm{d}}$, A.-K. Overkemping ${ }^{\mathrm{d}}$,

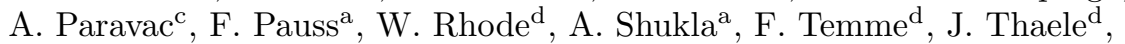 \\ S. Toscano ${ }^{\mathrm{b}}$, P. Vogler ${ }^{\mathrm{a}}$, R. Walter ${ }^{\mathrm{b}}$, A. Wilbert ${ }^{\mathrm{c}}$ \\ ${ }^{a}$ ETH Zurich, Institute for Particle Physics \\ Otto-Stern-Weg 5, 8093 Zurich, Switzerland \\ ${ }^{b}$ University of Geneva, ISDC Data Center for Astrophysics \\ Chemin d'Ecogia 16, 1290 Versoix, Switzerland \\ ${ }^{c}$ Universität Würzburg, Institute for Theoretical Physics and Astrophysics \\ Emil-Fischer-Str. 31, 97074 Würzburg, Germany \\ ${ }^{d}$ TU Dortmund, Experimental Physics 5 \\ Otto-Hahn-Str. 4, 44221 Dortmund, Germany
}

\begin{abstract}
Imaging Atmospheric Cherenkov Telescopes (IACTs) need imaging optics with large apertures and high image intensities to map the faint Cherenkov light emitted from cosmic ray air showers onto their image sensors. Segmented reflectors fulfill these needs, and composed from mass production mirror facets they are inexpensive and lightweight. However, as the overall image is a superposition of the individual facet images, alignment remains a challenge. Here we present a simple, yet extendable method, to align a segmented reflector using its Bokeh. Bokeh alignment does not need a star or good weather nights but can be done even during daytime. Bokeh alignment optimizes the facet orientations by comparing the segmented reflectors Bokeh to a predefined template. The optimal Bokeh template is highly constricted by the reflector's aperture and is easy accessible. The Bokeh is observed using the out of focus image of a near by point like light source in a distance of about 10 focal lengths. We introduce Bokeh alignment on segmented reflectors and demonstrate it on the First Geiger-mode Avalanche Cherenkov Telescope (FACT) on La Palma, Spain.
\end{abstract}

*S. A. Mueller sebmuell@phys.ethz.ch

Preprint submitted to Astroparticle Physics

May 23, 2016 
Keywords: Mirror alignment, point spread function, segmented reflector

\section{Introduction}

The IACT technique and its large effective area has opened the very high energy $\gamma$ ray sky to astronomy. Almost [1] all former [2, 3], current [4, 5, 6, 7, 8, and future IACTs [9, 10] make use of segmented imaging reflectors with apertures up to $614 \mathrm{~m}^{2}$ [6]. Segmented reflector facets can be mass produced inexpensively with an acceptable image quality. Facets are much lighter than a monolithic mirror, and thus allow for very fast telescope repositioning e.g. for $\gamma$ ray burst hunting.

However, there is one challenge to segmented reflectors. This is the task of manipulating the mirror facet orientations and positions to improve the image quality, also known as alignment. Alignment needs to be done not only during installation but also in case of repair and replacement of facets. To find the few $\gamma$ ray induced events in the far more numerous class of hadronic cosmic ray induced events, the air shower records are analyzed for geometrical properties. This makes imaging quality and alignment important for an IACT.

\section{Current methods}

To tackle the challenge of alignment, several approaches are in use. We can summarize them in three categories. First, there is the $2 f$ alignment, which is simple and does neither need star light nor dark nights [3, 8, 11. However, $2 f$ alignment is geometrically very restricted, which makes e.g. reaching the inner facets, that might be shadowed by the image sensor housing, a challenge. A second class of alignment methods uses remote controllable orientation actuators on the mirror facets and the live image of a star on a dedicated screen 6, 12. The screen is best positioned in the reflector's focal plane, which might conflict with the image sensor position. Since the mirror actuation needs to be used extensively to overcome the PSF facet ambiguity, these methods do not scale to well with the number of facets. Also a lot of custom hardware is needed. Furthermore one gains no additional information about the individual facet surfaces. Most importantly this method needs a bright star and can only tolerate faint clouds. Only if the image sensor distance can be altered or the out of focus effect can be corrected for differently, then a close by artificial light 
source can be used instead of a star. Third, there are methods based on the ideas of SCCAN [13, which track a star and observes the reflections of the facets on a camera located in the reflector's focal point [14, 15]. However, this method is also complex, and needs a bright star. Without a second camera, the method also favors clear nights [15. Although the mounting of the camera in the reflector's focal point can be overcome with a $45^{\circ}$ mirror, no SCCAN based IACT alignment implementation [14, 15] is mounted permanently yet.

In this paper we present a new method, that overcomes most of these limitations. We call it Bokeh alignment. This novel method is less geometrically restricted than the $2 f$ method and has fewer problems regarding shadowing. It is a simple method, which does not need a lot of custom hardware or software. Yet it gives information about individual mirror facet surfaces. Bokeh alignment does not need equipment to be installed in the reflector's focal point or focal plane. Finally it can be done anytime, even during the day.

We will first introduce the Bokeh and the Depth of Field on general, thin imaging systems. Second, we will discuss the Bokeh in the special case of segmented reflectors and its close relation to alignment. Third, we will present how to align a segmented reflector using Bokeh alignment without star light or dark nights. Finally, we will show the most simple flavor of Bokeh alignment, done on the First Geiger-mode Avalanche Cherenkov Telescope (FACT) in a step by step manner.

\section{Depth of Field and the Bokeh}

All real imaging optics have a non zero aperture, i.e. the aperture diameter $D$ is greater zero. Furthermore, all non zero aperture imaging optics have the effect of Depth of Field. The Depth of Field makes the image of objects look blurred on the image sensor in case these objects are out of focus. This blurring is described by the Bokeh function $B$ of the imaging system. Bokeh is best known in photography where it is used to achieve certain image compositions [16. The Depth of Field blurring has also been discussed for IACTs [17, 18, 19, in particular in the context of image and object distances and its impact on the air shower image analysis.

\subsection{Bokeh on thin imaging optics}

The idealized thin imaging optic bends the incoming light only in its aperture plane. In practice, this is a good approximation for lenses and reflectors with a 
large $F$-number, $F=f / D$ where $f$ is the optic's focal length. On thin imaging optics, the Bokeh effect is described by the geometry of the thin lens equation

$$
\frac{1}{f}=\frac{1}{b}+\frac{1}{g}
$$

together with an image sensor plane located at distance $d$, see Figure 1 The thin lens equation defines the image distance $b$ where an image sensor must be placed, on an imaging system with focal length $f$, in order to obtain a sharp image of an object in distance $g$. In contrast to this in focus situation, the image sensor can be placed elsewhere, e.g. in $d \neq b$, and the image of the object in distance $g$ becomes out of focus, see Figure 1 .

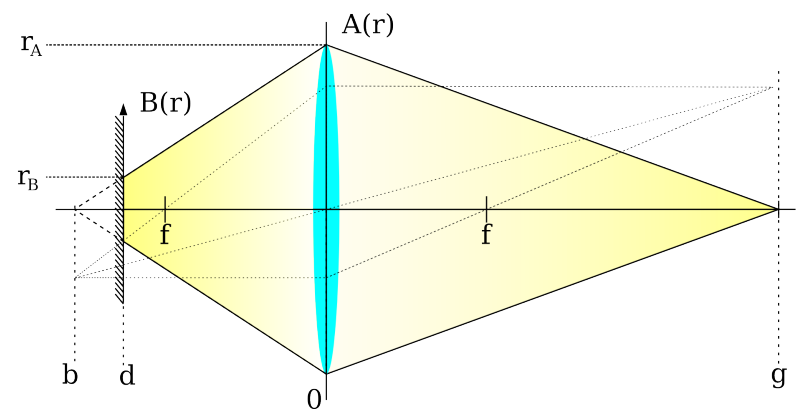

Figure 1: A thin lens and an image sensor, facing an object where the image distance $b$ is not equal to the image sensor distance $d, b \neq d$. This is the out of focus situation.

In this case, the point like object in distance $g$ is not mapped sharply onto the image sensor plane in distance $d$ and becomes blurred. This intensity distribution of the object on the image sensor is called Bokeh function $B(r, \varphi)$. In the case of thin imaging optics, this Bokeh $B(r, \varphi)$ is equal to the aperture function $A(r, \varphi)$ up to a scaling factor in size $\frac{r_{B}}{r_{A}}$

$$
B(r, \varphi)=\frac{r_{B}}{r_{A}} A(r, \varphi)
$$

The Aperture function $A(r, \varphi)$ is a two dimensional encoding of the transmission or reflection coefficient of the aperture. Here $\varphi$ and $r$ are the polar coordinates orthogonal to the optical axis. Given Figure 1 we use the intercept theorem and 
Equation 1 to find the scaling between $B$ and $A$

$$
\frac{r_{B}}{r_{A}}=1-d\left(\frac{1}{f}-\frac{1}{g}\right) .
$$

\subsection{How to observe the Bokeh of an imaging optic}

The image of an object plane in distance $g$, which is mapped onto an out of focus image sensor in $d$, is the result of a convolution of the object plane with the imaging system's Bokeh function $B(r, \varphi)$. When there is only a single $\delta$-distribution like feature in the object plane, the image shows the convolution kernel itself, i.e. the Bokeh function $B(r, \varphi)$. Therefore, to get an imaging optic's Bokeh $B$ one only needs to acquire images of a point like light source in an out of focus configuration.

\subsection{The Bokeh of segmented imaging reflectors}

On segmented imaging reflectors the aperture function $A$ has special features because of the shape of the mirror facets and the gaps in-between them. Since the Bokeh function $B$ is a scaling of the aperture function $A$, see Equation 3 , it will also have these special features. However, this is only true when the individual facets are well aligned.

The Figures 2 and 3 show both the same well aligned segmented imaging reflector. The difference in the Figures is the image sensor position, which is an in focus set-up in Figure 2 and an out of focus set-up in Figure 3 . Figure 4 shows that $B$ strongly depends on the alignment state of the reflector. The

light bundles of the individual facets might overlap, and thus lead to obvious mismatches between $A$ and $B$. 


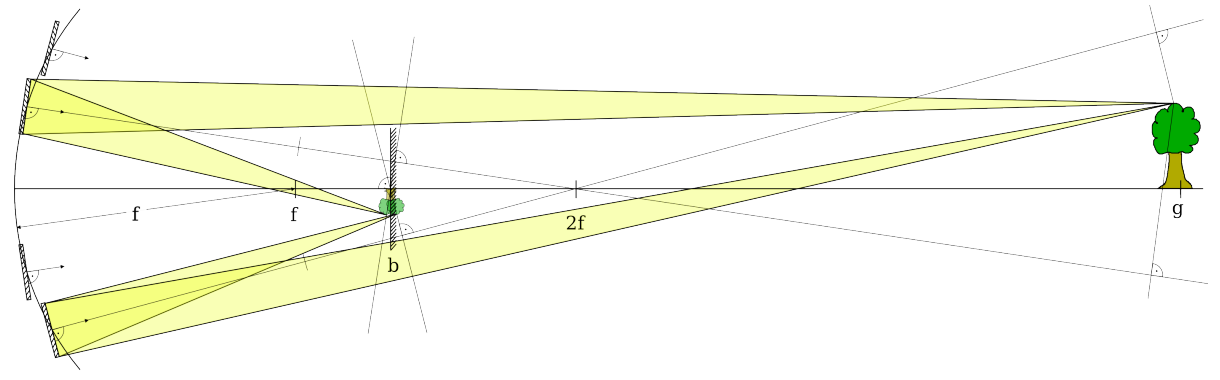

Figure 2: A well aligned segmented imaging reflector in Davies Cotton [20] configuration is observing a tree in distance $g$. Only two of the four mirror light bundles are highlighted. The image sensor is placed in distance $d=b$, which corresponds to the in focus situation of Equation 1

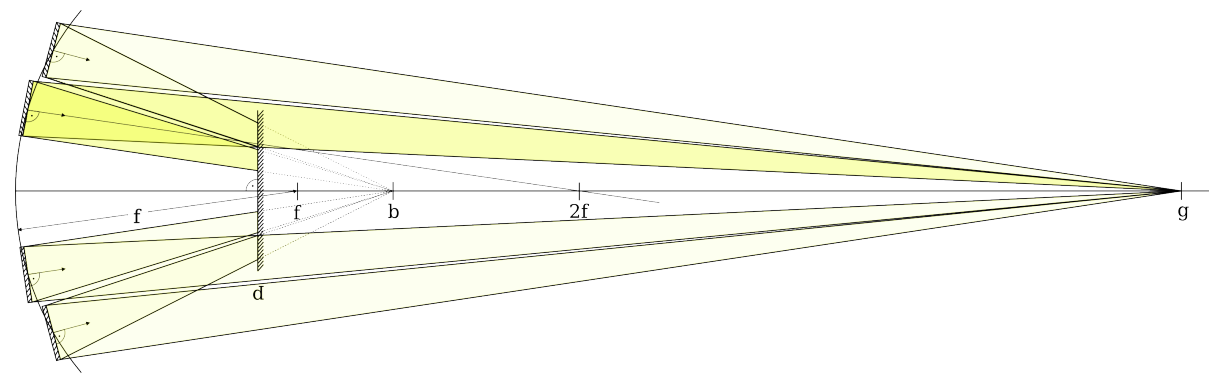

Figure 3: The segmented imaging reflector as in Figure 2. A point like light source shines into the reflector while the image sensor is out of focus in $d \neq b$. The image of the light source reveals the Bokeh $B$, see Section 3.2 All mirror light bundles are highlighted. 


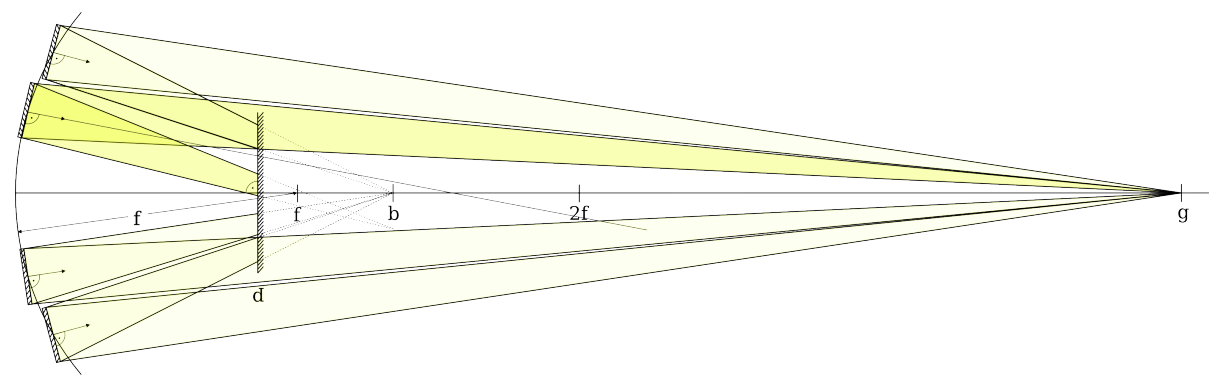

Figure 4: The segmented imaging reflector as in Figure 3 but now the second facet from the top is misaligned. $B$ does no longer match the scaled $A$.

\subsection{The $2 f$ method is a special case of Bokeh alignment}

In $2 f$ alignment, the light source or Bokeh lamp is placed in $g=2 f$ and the image sensor to observe the mirror facet reflections is also placed in $d=2 f$. According to Equation 1, this is the in focus configuration and according to Equation 3 the Bokeh ratio is $\frac{r_{B}}{r_{A}}=0$. Therefore the observed Bokeh $B$ becomes a point like $r_{B}=0$ spot observed during the $2 f$ alignment.

\section{How to perform Bokeh alignment on a segmented reflector}

We make use of the Bokeh's dependence on the alignment state of a segmented reflector to formulate an alignment procedure. The facets can be aligned by matching the reflector's actual Bokeh $B$ to an ideal Bokeh template $B_{\text {template, }}$ which is the Bokeh of the well aligned reflector. In case of thin imaging systems, Equation 2 shows us a simple way to obtain such a template $B_{\text {template }}$. We just have to know the aperture $A$ of the segmented reflector to calculate $B_{\text {template }}$ using the scaling Equation 3 . On non-thin imaging systems one can improve the $B_{\text {template }}$ by using different techniques, see Section 6.1. In general the following steps have to be done.

- Create a Bokeh template $B_{\text {template }}$ of the well aligned segmented reflector.

- Place a screen on the reflector in distance $d$ to project the reflector's Bokeh $B$ on. The screen should not cause additional shadowing, i.e. it should not be bigger than the image sensor housing of the telescope.

- Point the telescope to a point like light source in distance $g$. We call this light source Bokeh lamp. 
- Observe the projected Bokeh $B$ on the screen and compare it to the template $B_{\text {template }}$.

- Align each facet to make its own $B$ on the screen overlap with its $B_{\text {template. }}$

- When the reflector's Bokeh $B$ matches $B_{\text {template }}$, the reflector is aligned.

\section{The Bokeh alignment of FACT}

We performed Bokeh alignment on FACT in May 2014 in the simple way

explained in Section 4. It should be noted that for the Bokeh alignment of FACT, not a single line of computer program was written. Further, the whole procedure, including preparation and hardware construction, took only a single day. All the tools used were:

- A Bokeh lamp, $3 \mathrm{~W}$ LED

- A desktop printer

- A consumer image processing tool, GIMP on gnu-Linux

- A consumer photo camera, Nikon DSLR with telephoto lens

- A laser distance meter

- Water levels and laser cross line levels

Table 1 and Figure 5 show the basic properties of FACT's imaging system. 


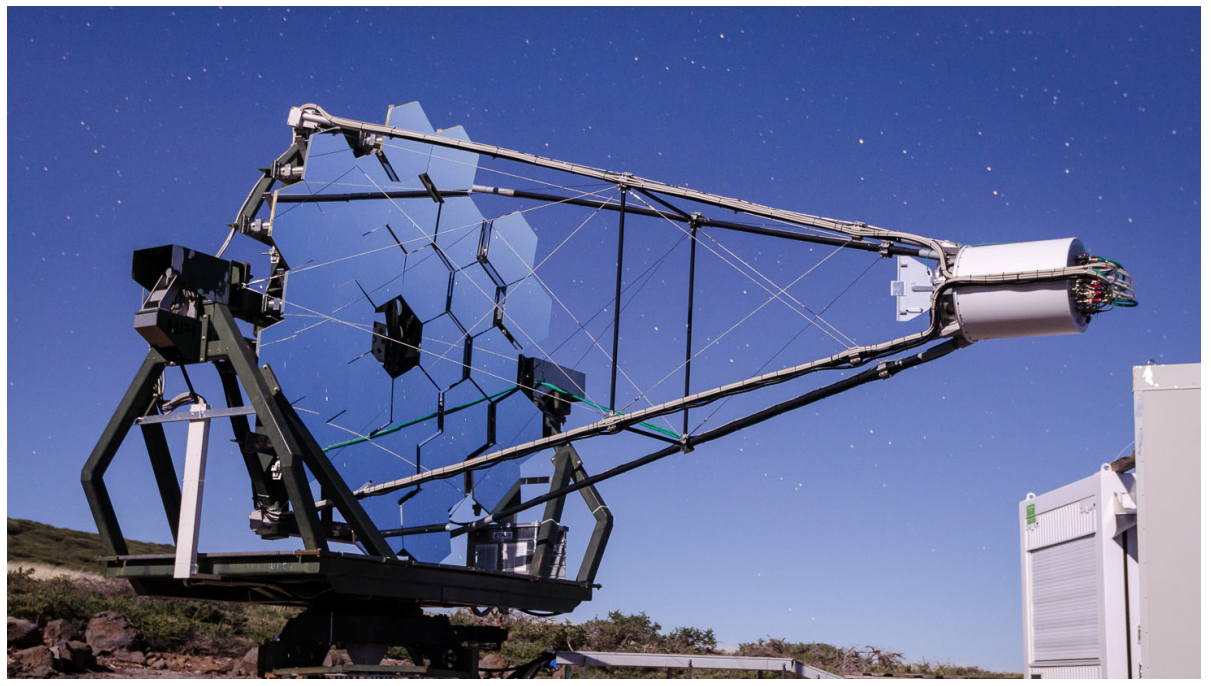

Figure 5: FACT is located on Canary island La Palma, Spain. It inherited its mount and the mirror facets from HEGRA 21. While pioneering silicon photomultipliers for IACTs, FACT is monitoring $\gamma$ ray bright Blazars such as Mrk 421 and Mrk 501. Photograph by Thomas Krähenbühl.

\begin{tabular}{lr}
\hline focal length $f$ & $4.889 \mathrm{~m}$ \\
number of facets & 30 \\
facet mounting & manual adjustment on tripod \\
reflector geometry along optical axis & $1 / 2$ Davies Cotton $+1 / 2$ parabola \\
reflector area $A$ & $9.51 \mathrm{~m}^{2}$ \\
effective reflector area $A_{\text {eff }}$ & $8.80 \mathrm{~m}^{2}$ \\
effective aperture diameter $D_{\text {eff }}$ & $3.35 \mathrm{~m}$ \\
maximum aperture diameter $D_{\max }$ & $3.93 \mathrm{~m}$ \\
effective F-number, $f / D_{\text {eff }}$ & 1.46 \\
F-number, $f / D_{\text {max }}$ & 1.25 \\
image sensor diameter, FoV & $0.39 \mathrm{~m}, 4.5^{\circ} \mathrm{deg}$ \\
\hline
\end{tabular}

Table 1: Basic imaging reflector properties of FACT

\subsection{Creating the Bokeh template $B_{\text {template }}$ for the well aligned FACT}

First we need to know the Bokeh $B_{\text {template }}$ of the well aligned FACT. We assume that FACT's imaging system is thin, i.e. that the methods from Chapter 3.1 are applicable. It is important to note, that FACT's reflector is neither thin 
in Davies Cotton nor in parabolic configuration. In Section 6.1 we extend the method to non thin imaging systems. For this first demonstration however, we restricted ourselves to the thin lens approximation, for the sake of simplicity. Now we can, according to Equation 2, use the aperture function $A$ to get the

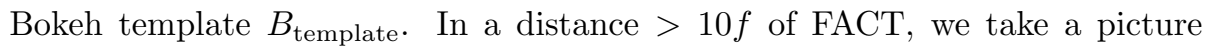
of the reflector's aperture $A$ with a telephoto lens for minimal distortions and measure $A$ with maximum contrast, see the red part of Figure 6.

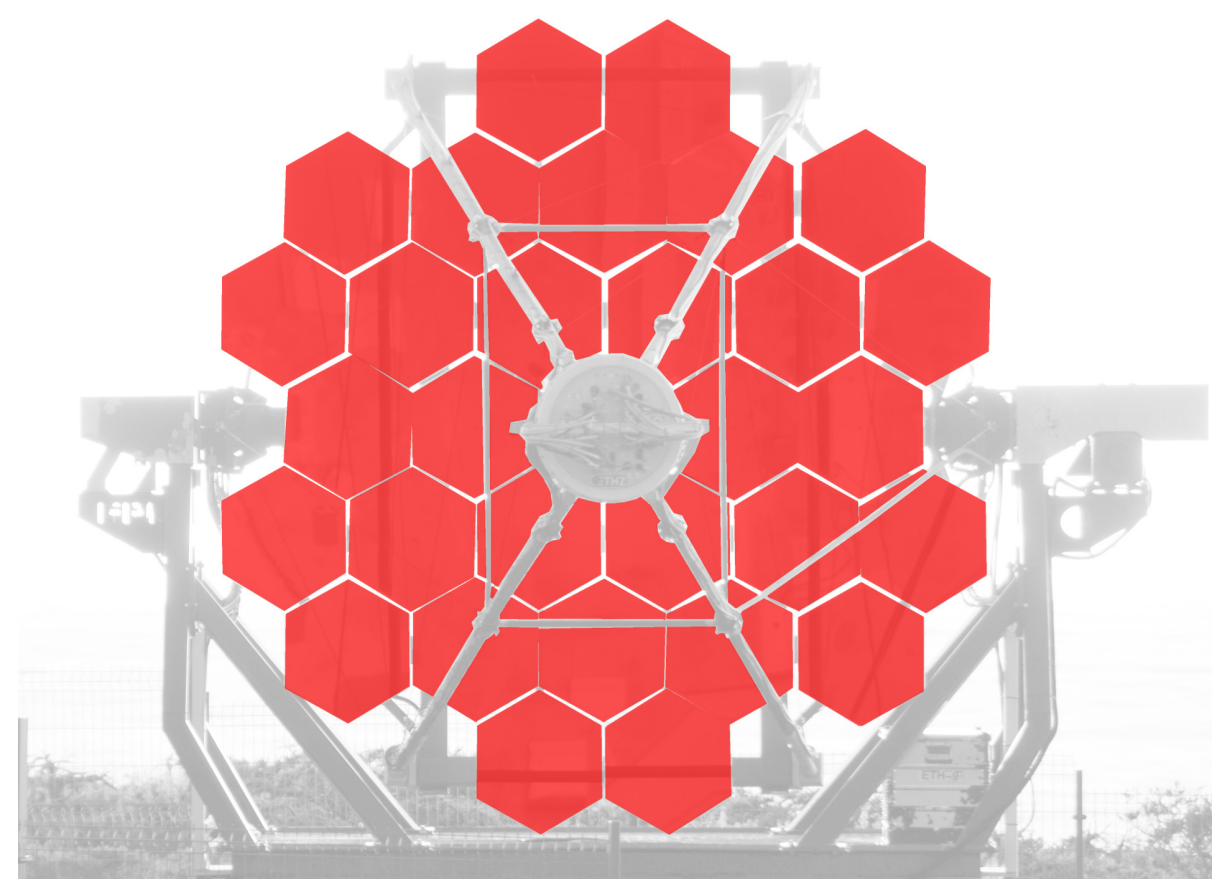

Figure 6: The aperture function $A$ in red, extracted from the aperture photograph.

\subsection{Creating the Bokeh template screen}

The Bokeh template $B_{\text {template }}$ is printed on paper and glued onto a flat shield. This template screen serves as temporary imaging screen, see Figure 7. The size of the screen $r_{B}$ is chosen such that the screen does not create additional shadowing on the reflector. The screen is mounted on the telescope in distance $d$ w.r.t. the reflector, which is $10 \mathrm{~cm}$ on top of FACT's image sensor. The Bokeh parameters of the alignment of FACT are shown in Table 2 


\begin{tabular}{lr}
\hline Aperture radius $r_{A}$ & $1.633 \mathrm{~m}$ \\
Bokeh radius $r_{B}$ & $0.190 \mathrm{~m}$ \\
Bokeh scaling $\frac{r_{B}}{r_{A}}$ & 0.1164 \\
$B_{\text {template distance } d}$ & $4.789 \mathrm{~m}$ \\
Bokeh lamp distance $g$ & $49.939 \mathrm{~m}$ \\
\hline
\end{tabular}

Table 2: Bokeh parameters for the alignment of FACT. Given $f$ and $r_{A}$ from FACT's design, we chose $r_{B}$ and $d$ to fit our needs. Finally, we calculate $g$ according to Equation 3

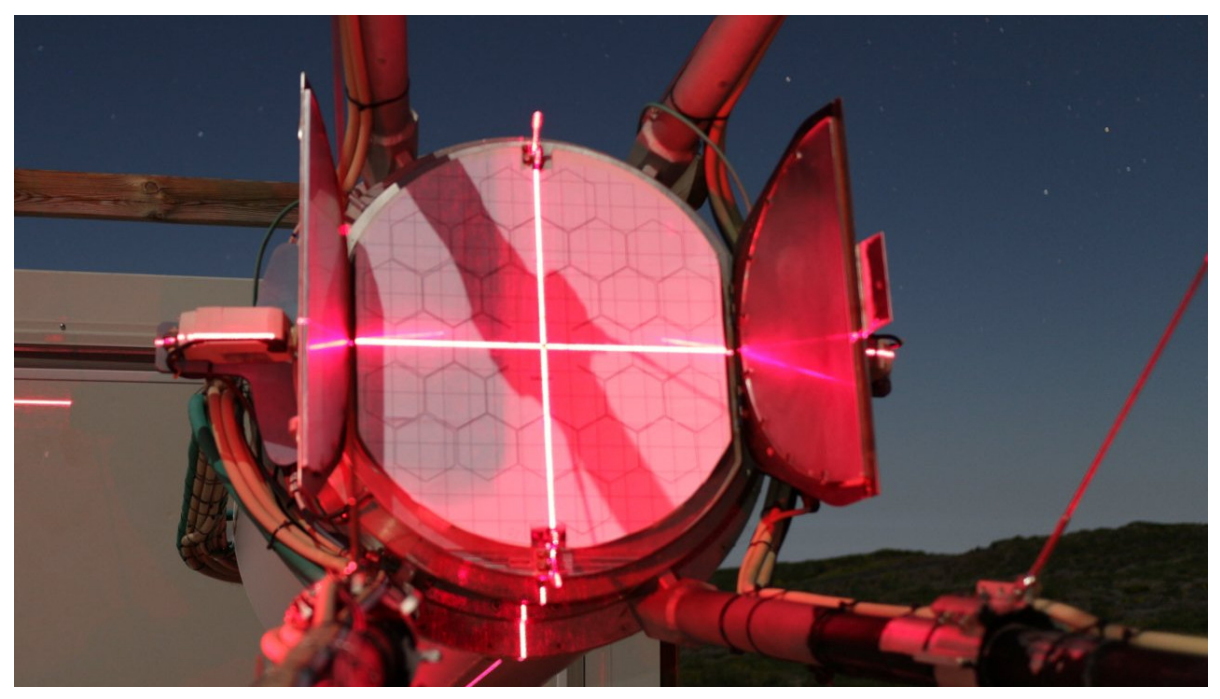

Figure 7: Mounting and positioning the $B_{\text {template }}$ screen in front of FACT's image sensor using the laser cross line level.

\subsection{Observing FACT's Bokeh}

To observe the Bokeh $B$ from the currently misaligned reflector, we point the telescope to our Bokeh lamp, see Figure 8, Our reflector then maps the actual $B$ onto the $B_{\text {template }}$ screen. For crosscheck, the single mirror facet's $B$ now should have the same size as the individual facet templates in $B_{\text {template }}$.

\subsection{Aligning FACT using its Bokeh}

We reorient each mirror facet to make its individual $B$ overlap the corresponding one in $B_{\text {template }}$. When the overlap is maximized, the alignment is 
done. The mirror joints are operated manually while watching the $B_{\text {template }}$ screen and the $B$ on it. Figures 8,9 and 10 show the Bokeh alignment of FACT during, before, and after the alignment.

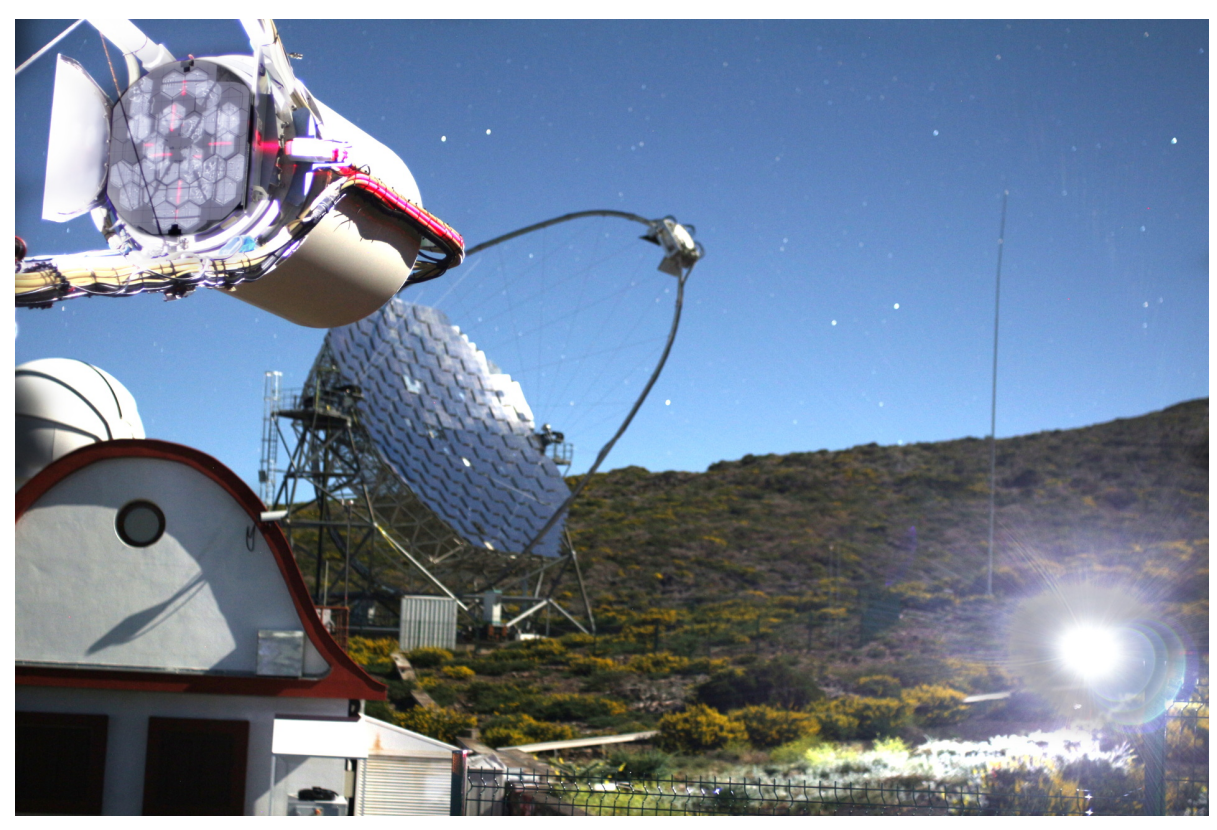

Figure 8: In the upper left we see FACT's sensor housing and the Bokeh template screen on top of the imaging sensor. In the lower right part we see the Bokeh lamp. The lower half of

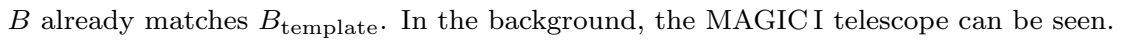



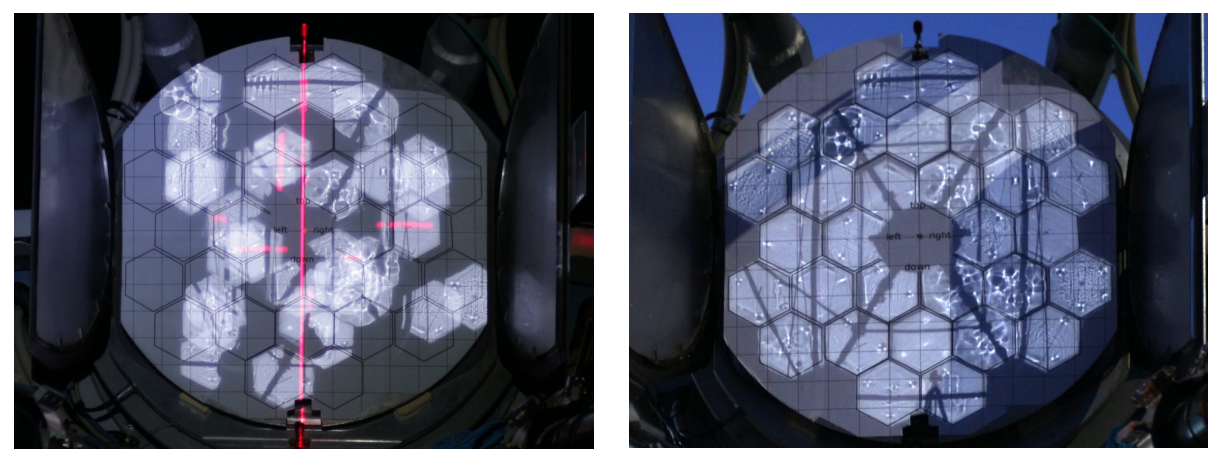

Figure 9: The initial Bokeh $B$ on FACT before alignment. It looks nothing like the template.

Figure 10: The Bokeh $B$ after finishing alignment. We finished alignment in late dawn. $B$ now matches the $B_{\text {template}}$, compare Figure 6

\subsection{Investigating individual facets using Bokeh}

In the same way as the overall Bokeh of a segmented reflector tells about the alignment sate of its facets, the individual facet Bokeh tells about the properties of the facet's surface. The Figures 11 and 12 show close up sections from Figure 10. Different types of surface distortions can be found. For example on FACT, the Bokeh reveals that all mirror facets have surface distortions at their tripod mounts. In contrast to the more advanced Phase Measuring Deflectometry (PMD) technique 22, a facet's Bokeh gives only limited information to reconstruct the facet's surface normals. But the advantage of the Bokeh method is its simple execution so that facet deformations, e.g. due to aging, might be recognized on a daily basis while the facets are installed on the telescope. Individual facet surface deformation information can not be deduced easily from a PSF image, where all individual facet PSFs are stacked on top of each other.

\subsection{Resulting Imaging performance on FACT}

FACT was initially aligned in 2011 using the $2 f$ method [8]. The PSF achieved in the $2 f$ method was in use until May 2014, and is shown in Figure 13. This PSF lead in particular to the characterization of the $\gamma$ ray emission from the Crab nebula 23]. In May 2014 the reflector geometry was reconfigured to form a hybrid of Davies Cotton and parabola in order to improve the time resolution [24] of the reflector. After this reconfiguration, FACT was unusable 


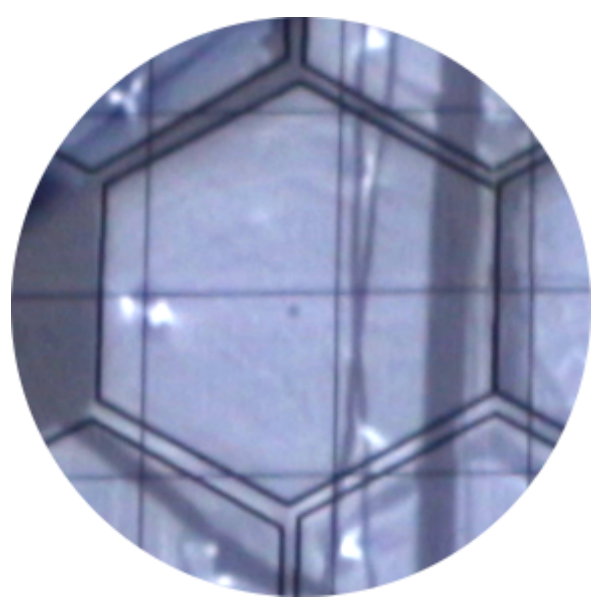

Figure 11: A homogeneous facet Bokeh. 4th row from top, 1st from left in figure 10

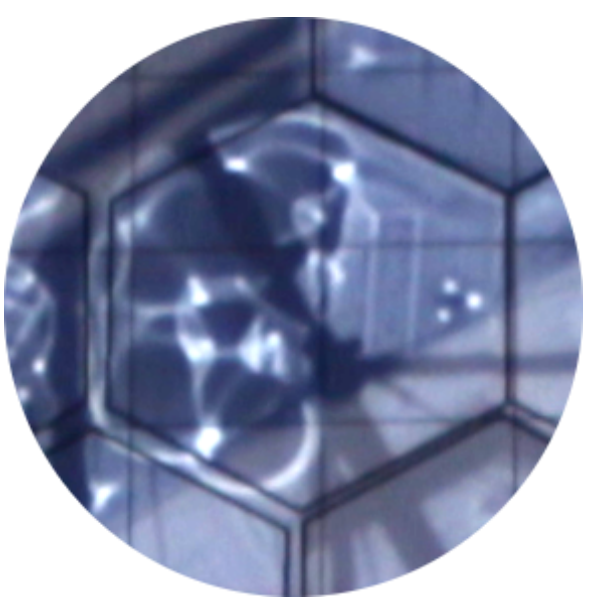

Figure 12: A partially distorted facet Bokeh. 2nd row from top, 2nd from left in Figure 10

\begin{tabular}{lr} 
Reflector state & $A_{\sigma}\left[\operatorname{arcmin}^{2}\right]$ \\
\hline before reconfiguration & 62.0 \\
after reconfiguration & too large to be recorded \\
after Bokeh alignment & 65.5 \\
\hline
\end{tabular}

Table 3: The FACT PSF $1 \sigma$ area before and after the reflector reconfiguration and Bokeh alignment, see Figures 13 and 14

since every facet was arbitrarily oriented. We performed the realignment using the novel Bokeh method. The new PSF after Bokeh alignment is shown in Figure 14. Bokeh alignment achieved a PSF similar in size to the one before the reflector reconfiguration. We did one Bokeh alignment iteration in one day. The PSF images 13 and 14 are recorded in the reflector's focal plane using a $6 \times 6 \mathrm{~cm}^{2}$ radiometrically calibrated image sensor while FACT is tracking the star Arcturus. This dedicated image sensor was made out of a vintage medium format camera (Hasselblad 6x6) and an industrial ccd camera. Mounted on FACT, this image sensor covers $0.75^{\circ} \times 0.75^{\circ}$. In both Figures 13 and 14 , an enclosure ellipse of the light intensity distribution is highlighted in red together with a blue FACT pixel aperture for size comparison. The PSF in Figure 14 is a bit blurred because of wind during the exposure. The enclosure ellipse areas $A_{\sigma}$ are compared in Table 3 If the time for more complex alignment methods 
can be afforded, Bokeh alignment is still efficient to be performed in advance as it was done later for the NAMOD alignment of FACT. [15].

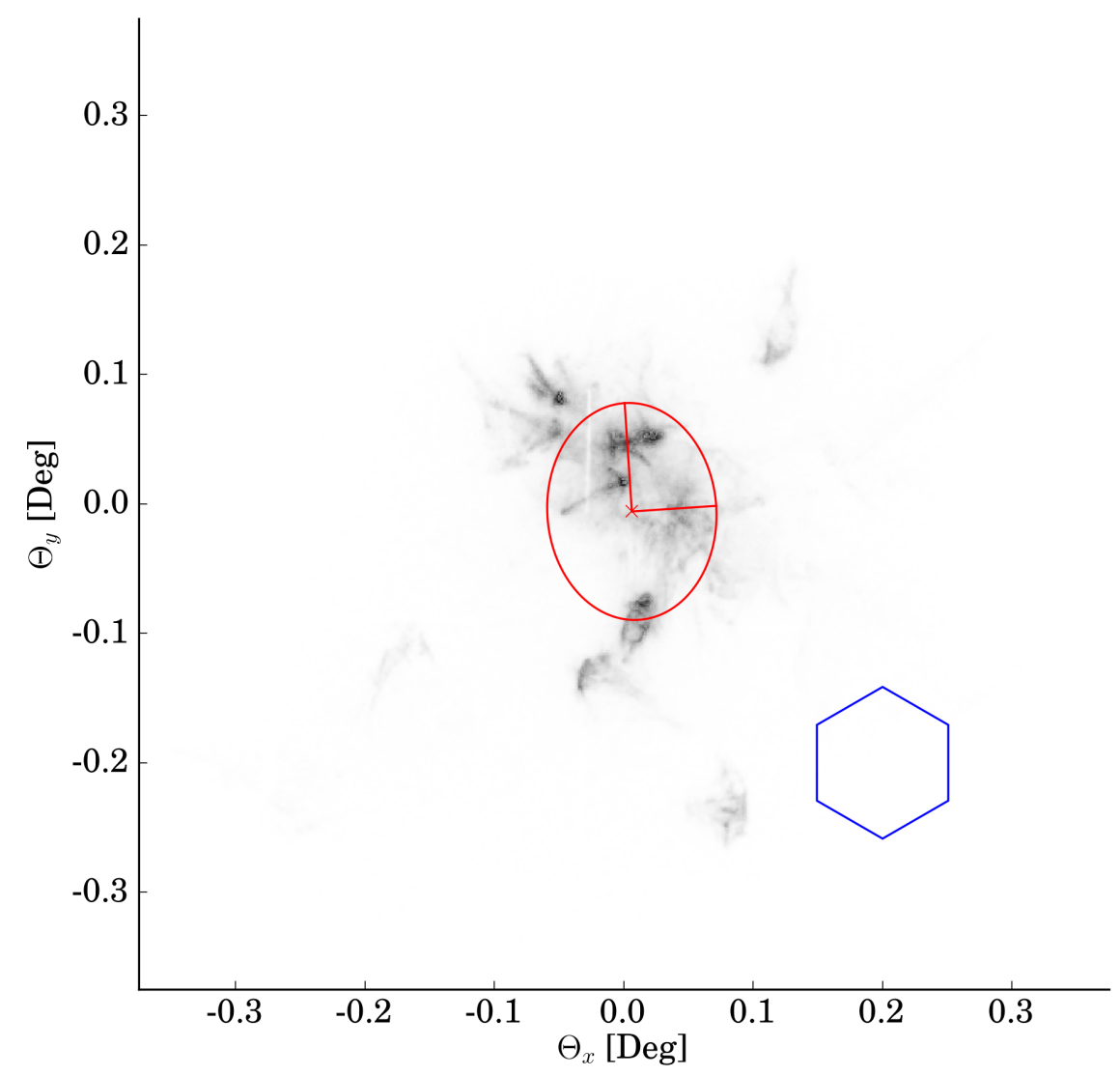

Figure 13: Image of star Arcturus in inverse color scale before the reflector reconfiguration. This PSF was achieved using the $2 f$ method in 2011. The $1 \sigma$ area $A_{\sigma}=62.0 \operatorname{arcmin}^{2}$ is shown in red. For comparison, a FACT pixel is shown in blue. 


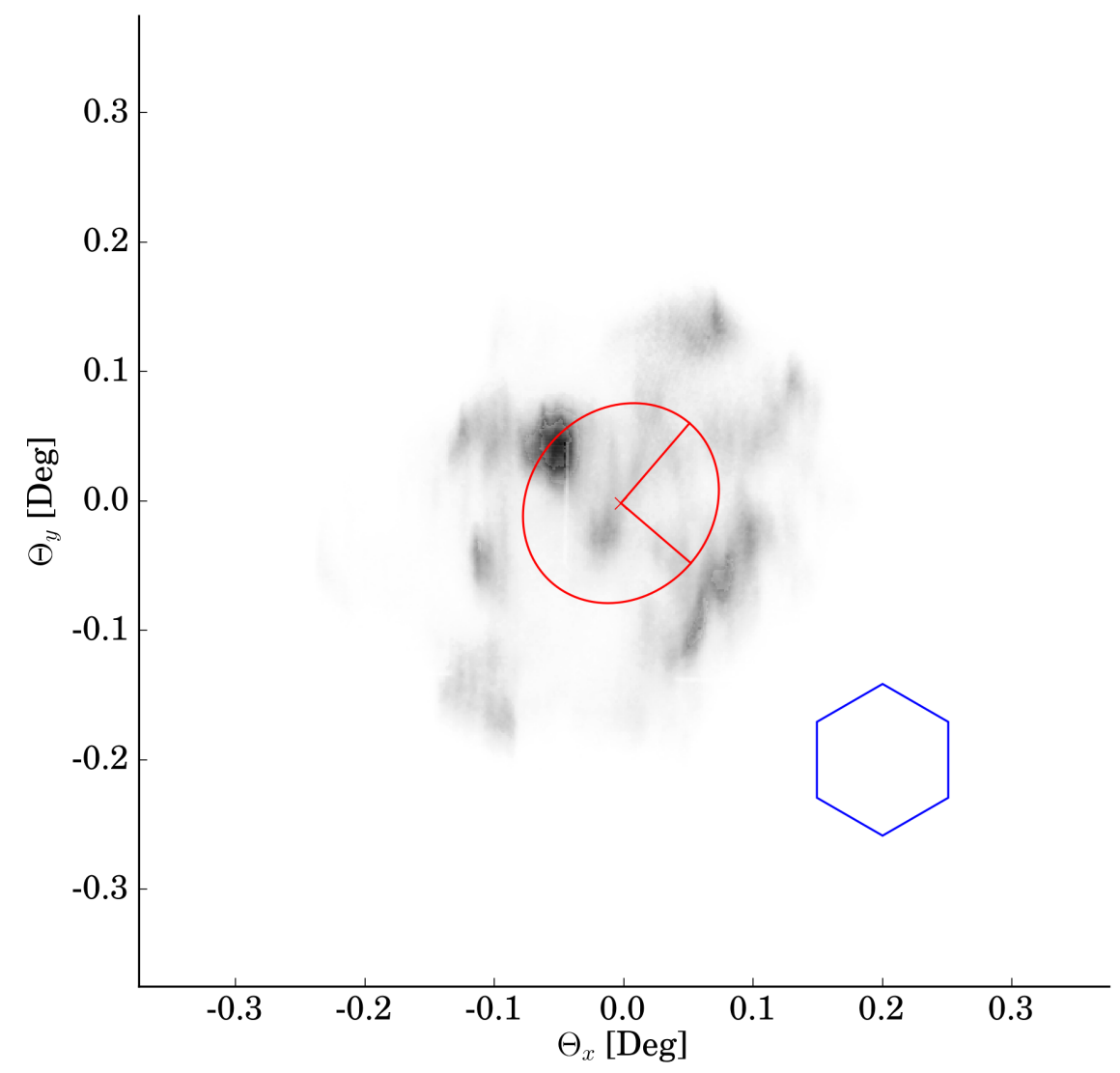

Figure 14: Image of star Arcturus in inverse color scale after reflector reconfiguration and Bokeh alignment. The $1 \sigma$ area $A_{\sigma}=65.5 \mathrm{arcmin}^{2}$ is shown in red. For comparison, a FACT pixel is shown in blue. Due to wind during the exposure, the PSF here does not show the same sharp features as they can be found in Figure 13

\section{Outlook}

In this outlook, we want to show what Bokeh alignment might look and feel like in the future, when one combines it with ray tracing simulations and computer vision [25]. 
6.1. Dropping the thin lens approximation $-B_{\text {template }}$ redone in ray tracing

Actual imaging systems are not thin and especially IACT optics are not thin with $f / D$ below 2. After the first Bokeh alignment of FACT, we used ray tracing to obtain a better approximation of $B_{\text {template }}$ based on FACT's actual non thin imaging reflector. Figure 15 shows the simulated light distribution on FACT's Bokeh screen for the configuration stated in Table 2. The actually used $B_{\text {template }}$, created using the thin lens approximation and shown in Figure

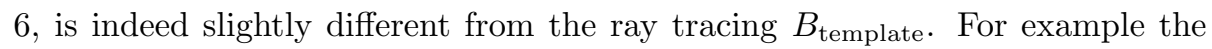
overlap of the facet edges is not predicted by the thin lens approximation. Using this $B_{\text {template }}$ will result in a better PSF and is currently investigated on the Cherenkov Telescope Array (CTA) 9] Medium Size Telescope (MST) prototype in Berlin Adlershof.

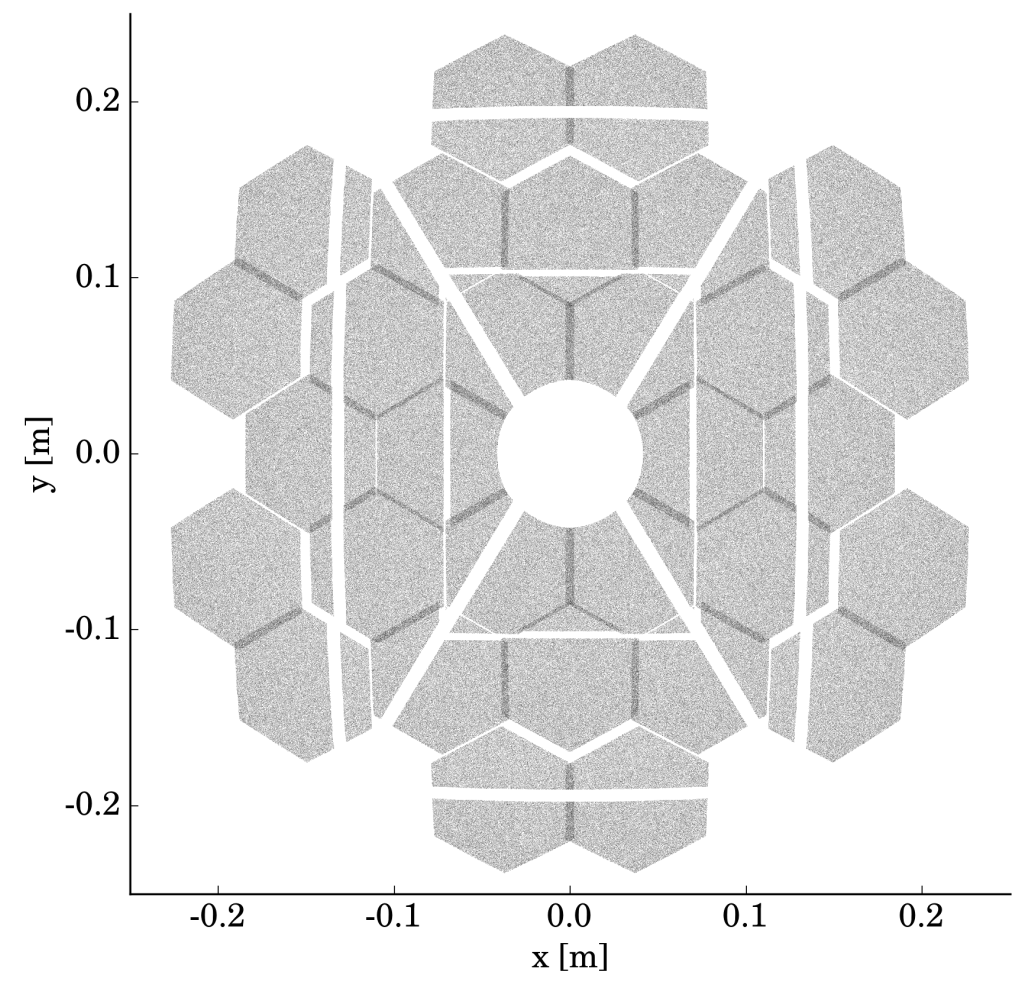

Figure 15: Simulated light distribution on the Bokeh screen of FACT, darker is more intense. Differences show up when comparing it to the thin lens approximated $B_{\text {template }}$ shown in Figure6 


\subsection{A $B_{\text {template }}$ for off axis observation}

To increase further the flexibility, one could create $B_{\text {template }}$ for an off axis configuration using ray tracing simulation. Here the Bokeh lamp is not restricted to the reflector's optical axis. This way the inner facets of the reflector can be reached more easily in case the telescope's sensor housing is shadowing them.

\subsection{Avoiding all facet ambiguity}

The problem of facet ambiguity is avoided when using a video projector instead of a simple lamp as Bokeh light source. This way the projector can address individual facets without illuminating the rest, which eliminates all ambiguity in the facet Bokeh relation.

\subsection{Observing $B$ very fast}

FACT's Bokeh alignment took several hours since we relied on pure eye hand coordination. However, the observation of $B$ can also be done very quickly since all misalignment information of the facets can be deduced from a single photograph of $B$.

\subsection{Observing $B$ during the day}

The observation capabilities of $B$ depend only of the ratio of Bokeh lamp light intensity to ambient light intensity on the Bokeh screen. For the night, dusk, and dawn, a $3 \mathrm{~W}$ Bokeh lamp worked for FACT but during the day, a

more powerful Bokeh lamp is needed. We tested with a consumer flash light on FACT and have been able to record $B$ in direct sunlight.

\subsection{Alignment for all telescope elevations}

On large telescopes gravitational slump effects the alignment depending on the elevation. Since a Bokeh flash light is small it can be lifted to desired elevations rather easy using a floating balloon, fastened to the ground, or a multi copter drone. Precise positioning of the Bokeh lamp is not crucial when the off axis Bokeh template is calculated and the lamp's position is reconstructed accurately. 


\subsection{Next level Bokeh alignment}

The lid of the telescope's image sensor will serve as Bokeh screen and will therefore be matt white. A flying drone is given a remote controlled flash light as Bokeh lamp. Additionally, drone cameras are mounted on the edge of the reflectors in order to reconstruct stereoscopically the position of the drone. A lid camera, located in the reflector dish, is observing the lid screen. A computer vision based Bokeh program reads out the lid camera, and the drone position. During dusk, the drone is started. Following the drone, the telescopes are moved to typical observation elevations. The Bokeh flash light is fired by the Bokeh program and the resulting Bokeh on the lid screen is recorded by the lid camera. Now the Bokeh program feeds the relative orientation of the drone and the telescope to a ray tracing simulation. The ray tracing simulation then returns the $B_{\text {template }}$ for this particular configuration as proposed in Section 6.1 and 6.2 From the deviations in between $B_{\text {template }}$ and $B$, the Bokeh program calculates the correction manipulations for the facets and sends these instructions to the mirror facet orientation control system of the telescope.

In such a setup one can:

- align the telescope without star light, dark nights or good weather.

- align without any overlap with $\gamma$ ray observation time.

- reach an alignment accuracy that is not intrinsically limited by the thin lens approximation.

- acquire the facet orientations for any telescope elevation.

- have special $B_{\text {template }}$ for individual use cases.

- keep track of each facet surface conditions.

- align a whole array of IACTs with a single flying drone.

\section{Conclusion}

In a first demonstration we aligned the FACT IACT using Bokeh alignment in a single day and a single iteration from an useless state, after a reflector reconfiguration, to an operational state for $\gamma$ ray astronomy. Bokeh alignment is inexpensive, fast and more versatile than current methods for the alignment of a segmented reflector. Boke alignment is simple, and does not need a lot of custom 
hardware or software. Yet it gives information about individual mirror facet surfaces and does neither need star light nor dark nights to align a segmented reflector. Boke alignment could even be done during the day.

Therefore, we believe that Bokeh alignment is ideal to align a large number of Cherenkov telescopes, for instance the upcoming Cherenkov Telescope Array (CTA).

\section{Acknowledgments}

The important contributions from ETH Zurich grants ETH-10.08-2 and ETH-27.12-1 as well as the funding by the German BMBF (Verbundforschung Astro- und Astroteilchenphysik) are gratefully acknowledged. We are thankful for the very valuable contributions from E. Lorenz, D. Renker and G. Viertel during the early phase of the project. We thank the Instituto de Astrofisica de Canarias allowing us to operate the telescope at the Observatorio Roque de los Muchachos in La Palma, the Max-Planck-Institut fuer Physik for providing us with the mount of the former HEGRA CT 3 telescope, and the MAGIC collaboration for their support. We would also like to thank Karsten Schuhmann for discussions on how to address individual mirror facets with a projector and fruitful discussions on optics in general.

\section{References}

\section{References}

[1] T. Hara, T. Kifune, Y. Matsubara, Y. Mizumoto, Y. Muraki, S. Ogio, T. Suda, T. Tanimori, M. Teshima, T. Yoshikoshi, et al., A $3.8 \mathrm{~m}$ imaging Cherenkov telescope for the $\mathrm{TeV}$ gamma-ray astronomy collaboration between Japan and Australia, Nucl. Instrum. Meth. A 332 (1) (1993) 300309.

[2] D. A. Lewis, Optical characteristics of the Whipple observatory Tev gamma-ray imaging telescope, Experimental Astronomy 1 (4) (1990) 213226.

[3] A. Barrau, R. Bazer-Bachi, E. Beyer, H. Cabot, M. Cerutti, L. Chounet, G. Debiais, B. Degrange, H. Delchini, J. Denance, et al., The CAT imaging telescope for very-high-energy gamma-ray astronomy, Nucl. Instrum. Meth. A 416 (2) (1998) 278-292. 
[4] J. Holder, R. Atkins, H. Badran, G. Blaylock, S. Bradbury, J. Buckley, K. Byrum, D. Carter-Lewis, O. Celik, Y. Chow, et al., The first VERITAS telescope, Astroparticle Physics 25 (6) (2006) 391-401.

[5] K. Bernlöhr, O. Carrol, R. Cornils, S. Elfahem, P. Espigat, S. Gillessen, G. Heinzelmann, G. Hermann, W. Hofmann, D. Horns, et al., The optical system of the HESS imaging atmospheric Cherenkov telescopes. Part I: layout and components of the system, Astroparticle Physics 20 (2) (2003) $111-128$.

[6] R. Cornils, K. Bernlöhr, G. Heinzelmann, W. Hofmann, M. Panter, The optical system of the HESS II telescope, in: International Cosmic Ray Conference, Vol. 5, 2005, p. 171.

[7] C. Baixeras, M. Collaboration, et al., The MAGIC telescope, Nuclear Physics B-Proceedings Supplements 114 (2003) 247-252.

[8] H. Anderhub, M. Backes, A. Biland, V. Boccone, I. Braun, T. Bretz, J. Buß, F. Cadoux, V. Commichau, L. Djambazov, et al., Design and operation of FACT - the First G-APD Cherenkov Telescope, Journal of Instrumentation 8 (06) (2013) P06008.

[9] B. S. Acharya, M. Actis, T. Aghajani, G. Agnetta, J. Aguilar, F. Aharonian, M. Ajello, A. Akhperjanian, M. Alcubierre, J. Aleksić, et al., Introducing the CTA concept, Astroparticle Physics 43 (2013) 3-18.

[10] Y. Igor, et al., Imaging Camera and Hardware of Tunka-IACT, in: Proceedings of the 34th ICRC, Vol. 986, 2015.

[11] J. Toner, V. Acciari, A. Cesarini, G. Gillanders, D. Hanna, G. Kenny, J. Kildea, A. Mccann, M. Mccutcheon, M. Lang, et al., Bias alignment of the VERITAS telescopes, in: Proceedings of the 30th International Cosmic Ray Conference, Vol. 3, 2008, p. 1401.

[12] A. Biland, M. Garczarczyk, H. Anderhub, V. Danielyan, D. Hakobyan, E. Lorenz, R. Mirzoyan, M. Collaboration, The Active Mirror Control of the MAGIC Telescope, in: Proceedings of the 30th ICRC, Vol. 533, 2007, pp. 1353-1356.

[13] F. Arqueros, G. Ros, G. Elorza, D. Garcia-Pinto, A technique for the optical characterization of imaging air-Cherenkov telescopes, Astroparticle Physics 24 (1) (2005) 137-145. 
[14] A. McCann, D. Hanna, J. Kildea, M. McCutcheon, A new mirror alignment system for the VERITAS telescopes, Astroparticle Physics 32 (6) (2010) $325-329$.

[15] M. L. Ahnen, D. Baack, M. Balbo, M. Bergmann, A. Biland, et al., Normalized and Asynchronous Mirror Alignment for Cherenkov Telescopes, submitted to Astroparticle Physics.

[16] H. M. Merklinger, A technical view of Bokeh, Photo Techniques 18 (3).

[17] R. Mirzoyan, V. Fomin, A. Stepanian, On the optical design of VHE gamma ray imaging Cherenkov telescopes, Nucl. Instrum. Meth. A 373 (1) (1996) $153-158$.

[18] W. Hofmann, How to focus a Cherenkov telescope, Journal of Physics G: Nuclear and Particle Physics 27 (4) (2001) 933-939.

[19] K. Bernlöhr, A. Barnacka, Y. Becherini, O. B. Bigas, E. Carmona, P. Colin, G. Decerprit, F. Di Pierro, F. Dubois, C. Farnier, et al., Monte Carlo design studies for the Cherenkov Telescope Array, Astroparticle Physics 43 (2013) $171-188$.

[20] J. M. Davies, E. S. Cotton, Design of the Quartermaster Solar Furnace, Solar Energy 1 (2) (1957) 16-22.

[21] V. Fonseca, Status and Results from the HEGRA Air Shower Experiment, Astrophysics and space science 263 (1-4) (1998) 377-380.

[22] A. Schulz, R. Krobot, E. Olesch, C. Faber, F. Stinzing, C. Stegmann, G. Hausler, et al., Methods for the characterization of mirror facets for Imaging Atmospheric Cherenkov Tele-scopes, in: Proc. 32nd Int. Cosmic Ray Conf, Vol. 9, 2011, pp. 34-37.

[23] F.Temme, et al., FACT - First Energy Spectrum from a SiPM Cherenkov Telescope, in: Proceedings of the 34th ICRC, Vol. 707, 2015.

[24] M. Noethe, et al., FACT - Calibration of Imaging Atmospheric Cherenkov Telescopes with Muon Rings, in: Proceedings of the 34th ICRC, Vol. 733, 2015 .

[25] D. A. Forsyth, J. Ponce, Computer Vision - A Modern Approach, Pearson Education Inc., New Jersey U.S.A., 2003. 